\title{
Evidence of a unique inter-allelic epistatic interaction for seed coat color in pigeonpea [Cajanus cajan (L.) Millspaugh]
}

\author{
K. B. Saxena $\cdot$ R. K. Saxena $\cdot$ R. V. Kumar $\cdot$ \\ R. K. Varshney
}

Received: 5 October 2011 / Accepted: 21 December 2011/Published online: 4 January 2012

(C) The Author(s) 2012. This article is published with open access at Springerlink.com

\begin{abstract}
For enhancing productivity and profitability of pigeonpea (Cajanus cajan (L.) Millsp.) a hybrid breeding technology based on a cytoplasmic nuclear male-sterility (CMS) system was developed at International Crops Research Institute for the Semi-Arid Tropics (ICRISAT). Among the elite hybrids 'ICPH 2671' was most out-standing with 30-35\% yield advantage over local varieties. Despite its commercial release in 2010, this hybrid could not attract many growers due to its less preferred seed color. Both the parents (ICPA 2043 and ICPR 2671) of this hybrid and their crossed seeds had Greyed-orange [code no*: 167B/C (* The standard identification numbers of Royal Horticultural Society)] color. However, the commercial seeds produced on the hybrid plants surprisingly have Greyed-purple (code no: 183C) color that fetches relatively less profits. To understand the genetics of this phenomenon a cross combination was studied for seed color in $F_{1}, F_{2}$, and $B_{1} C_{1} F_{1}$ generations. The data suggested that three independent dominant genes controlled the expression of seed coat color. The observations further suggested the presence of two dominant genes $\left(\mathrm{Brsd}_{1}\right.$ and $\left.\mathrm{Brsd}_{2}\right)$ in the malesterile line 'ICPA 2043', 'ICPR 2671' and another
\end{abstract}

K. B. Saxena $(\bowtie) \cdot$ R. K. Saxena · R. V. Kumar . R. K. Varshney

International Crops Research Institute for the Semi-Arid

Tropics (ICRISAT), Patancheru 502 324,

Andhra Pradesh, India

e-mail: k.saxena@cgiar.org dominant gene $\left(\mathrm{Brsd}_{3}\right)$ in the restorer line. In the hybrid plants genes $\operatorname{Brsd}_{1}$ and $\mathrm{Brsd}_{2}$ act in complementation with the third gene $B r s d_{3}$, this also acts as a modifier. The presence of $B r s d_{3}$ gene in a genotype together with $B r s d_{1}$ and/or $B r s d_{2}$ produces a strong inter-allelic epistatic interactions resulting in the Greyed-purple (183C) seed color. It was further postulated that the genetic constitution of 'ICPA 2043' is $\mathrm{Brsd}_{1} \mathrm{Brsd}_{1} \mathrm{Brsd}_{2} \mathrm{Brsd}_{2} \mathrm{brsd}_{3} \mathrm{brsd}_{3}$, while the genotype of the restorer line 'ICPR 2671' is $\operatorname{brsd}_{1} \operatorname{brsd}_{1} \operatorname{brsd}_{2} \mathrm{brsd}_{2} \mathrm{Brsd}_{3} \mathrm{Brsd}_{3}$.

Keywords Gene interaction · Hybrids · Inheritance · Pigeonpea $\cdot$ Seed-coat color

\section{Introduction}

Pigeonpea [Cajanus cajan (L.) Millsp.] is an important food legume in a number of semi-arid tropical countries of Asia, Africa, and the Americas. Seed coat color in pigeonpea plays an important role in consumer preference and market acceptability. Although the color of decorticated dry split peas is independent of its seed coat color, the pigeonpea grains with Greyed-purple (183C) color are less preferred from commercial points of view. In global pigeonpea germplasm the natural variation for seed color is large (Remanandan 1990); but most of the landraces and released cultivars have Greyed-orange 
$(167 \mathrm{~B} / \mathrm{C})$ or white $(\mathrm{NN} 155 \mathrm{C})$ seeds. Information on the inheritance of seed coat color in pigeonpea is meager, lacks clarity, and often complex due to interactions among primary and modifiers genes (reviewed by Saxena and Sharma 1990).

In order to enhance productivity and profitability of pigeonpea, a hybrid breeding technology based on a CMS (Saxena et al. 2005) system was developed at ICRISAT (Saxena 2008). Among the pigeonpea hybrid combinations developed, a medium maturing hybrid 'ICPH 2671' was found out-standing in the on-farm trials with 30-35\% yield advantage over local varieties in India (Saxena 2010) and 12-53\% in Myanmar (Kyu et al. 2011). This hybrid performed well even under limited moisture conditions and demonstrated high levels of resistance to wilt and sterility mosaic diseases. However, despite high yield advantages, this hybrid could not attract many farmers as the traders offer $10-15 \%$ less price due to its Greyed-purple (183C) seed color. It was surprising to note that the seed coat colors of both the parents and that of cross-pollinated seed harvested from the malesterile plants were Greyed-orange $(167 \mathrm{~B} / \mathrm{C})$ but the commercial seeds produced on the hybrid plants had, unexpectedly, Greyed-purple (183C) seeds (Fig. 1); and this has puzzled the breeders. Therefore, a study was undertaken to understand the genetic systems controlling seed coat color in this hybrid.

\section{Materials and methods}

Hybrid 'ICPH 2671' was produced by crossing a medium-duration CMS line 'ICPA 2043' and a restorer line 'ICPR 2671'. Self-seeds of the restorer parent were sown in 2007 rainy season at ICRISAT Center, Patancheru, India, along with A and R-lines under a bee-proof nylon net and hand pollinations were done to produce crossed $(\mathrm{A} \times \mathrm{R})$ seeds. In 2008, the $F_{1}$ plants were selfed to produce $F_{2}$ seeds. These plants were also crossed to the female parent 'ICPA 2043' to produce $\mathrm{BC}_{1} \mathrm{~F}_{1}$ seeds. In 2009 rainy season, the seeds of the parents, $\mathrm{F}_{1}, \mathrm{~F}_{2}$, and $\mathrm{BC}_{1} \mathrm{~F}_{1}$ generations were grown inside a large bee-proof cage on ridges, spaced $75 \mathrm{~cm}$ apart. The intra-row spacing was kept at $25 \mathrm{~cm}$. The plot size for parents ( 2 rows), $F_{1}$ (10 rows) $\mathrm{F}_{2}$ (15 rows), and $\mathrm{BC}_{1} \mathrm{~F}_{1}$ (10 rows) were determined by the seed availability In each generation, seeds of all the individual plants were examined for their coat color

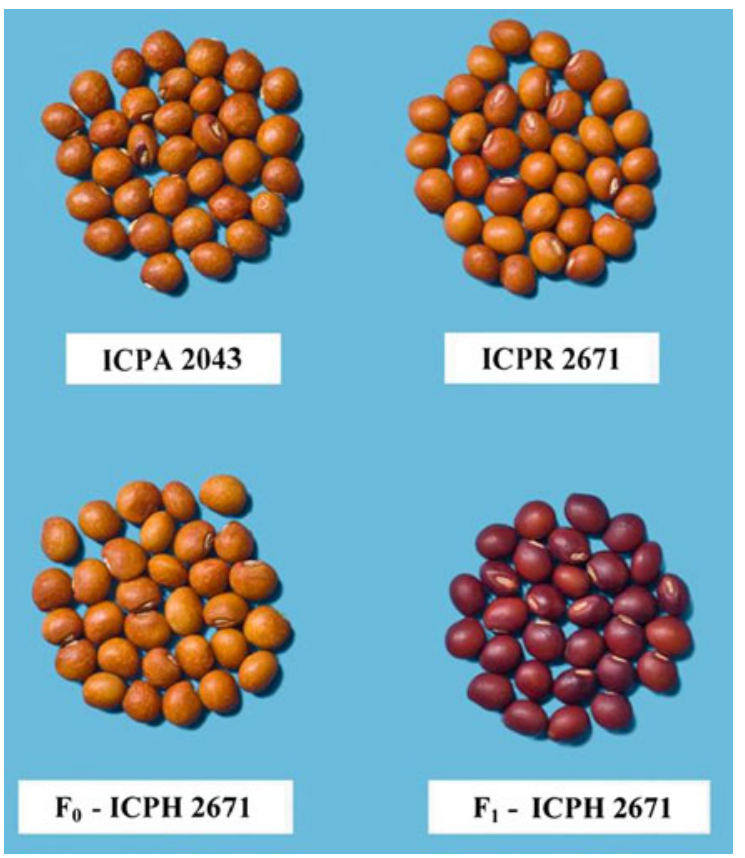

Fig. 1 Seed coat colors of the female parent ICPA 2043, male parent ICPR 2671, crossed seed harvested from the female parent, and the commercial seeds produced on $F_{1}$ plants of hybrid ICPH 2671

using the standard Identification numbers of Royal Horticultural Society, London (www.rhs.org.uk). The data recorded in each generation were subjected to Chi-square test to assess their goodness of fit to different phenotypic ratios.

\section{Results and discussion}

The color of crossed $(\mathrm{A} \times \mathrm{R})$ seed was Greyed-orange $(167 \mathrm{~B} / \mathrm{C})$ and similar to the parents. However, the seeds harvested from the $\mathrm{F}_{1}$ plants were of Greyedpurple (183C) (Fig. 1). The $\mathrm{F}_{2}$ population of this cross segregated for seed color; and out of 235 plants grown, 158 had Greyed-purple (183C) seeds and the remaining 77 plants produced the parental type Greyedorange $(167 \mathrm{~B} / \mathrm{C})$ seeds. This segregation fit well to the expected phenotypic ratio of 45 Greyed-purple (183C): 19 Greyed-orange $(167 \mathrm{~B} / \mathrm{C})(P=0.30)$ indicating involvement of three independent dominant genes. In the $\mathrm{BC}_{1} \mathrm{~F}_{1}$ generation, a total of 137 plants were studied and of these, 70 plants produced Greyedpurple (183C) seeds, while 67 plants had the parental type Greyed-orange (167B/C) seeds. This segregation 
fitted well to the expected ratio of 1 Greyed-purple (183C): 1 Greyed-orange $(167 \mathrm{~B} / \mathrm{C})$ seeds $(P=0.79)$, confirming the presence of a tri-genic system in governing seed color in ICPH 2671.

Based on the segregation patterns observed in the progenies of this cross, it is postulated that the genetic system controlling the seed color was determined by three independent dominant genes. Of these, two dominant genes $\left(\mathrm{Brsd}_{1}\right.$ and $\mathrm{Brsd}_{2}$ ) were present in the female parent, while the restorer line had only one dominant gene $\left(\mathrm{Brsd}_{3}\right)$ that was different from the other two genes. The presence of any one $\left(B r s d_{1}\right.$ or $B r s d_{2}$, ) or both the dominant genes together $\left(\operatorname{Brsd}_{1}+\operatorname{Brsd}_{2}\right)$ were responsible for producing Greyed-orange (167B/ C) colored seeds. Similarly, the third dominant gene $\left(B r s d_{3}\right)$, also produced Greyed-orange (167B/C) seeds, when present alone. The $\left(\mathrm{Brsd}_{3}\right)$ gene interacted with the other two dominant genes individually $\left(\operatorname{Brsd}_{1}\right.$ or $\left.B r s d_{2}\right)$ or together $\left(B r s d_{1}+B r s d_{2}\right)$ and produced the unique Greyed-purple (183C) seeds.

The $\mathrm{F}_{1}$ plants with expected genotype of Brsd $_{1} b r s d_{1}$ $\mathrm{Brsd}_{2} \mathrm{brsd}_{2} \mathrm{Brsd}_{3} \mathrm{brsd}_{3}$ produced Greyed-purple (183C) seeds due to interaction of $\mathrm{Brsd}_{3}$ with the other two dominant genes. As expected in $\mathrm{F}_{2}$ generation, all the three genes segregated independently and assorted randomly to yield phenotypic ratio of 49 Greyedpurple (183C): 15 Greyed-orange $(167 \mathrm{~B} / \mathrm{C})$. This phenotypic of Greyed-purple (183C) seeds involved three genotypes and their genotypic frequencies were $\mathrm{Brsd}_{1}-\mathrm{Brsd}_{2}-\mathrm{Brsd}_{3}$ (27/64), brsd $_{1}$ brsd $_{1} \quad \mathrm{Brsd}_{2}-$ $\operatorname{Brsd}_{3}(9 / 64)$, and Brsd $_{1}-$ brsd $_{2}$ brsd $_{2} \quad \mathrm{Brsd}_{3}(9 / 64)$. Similarly, the Greyed-orange (167B/C) seeded plants consisted of five different genotypes and their genotypic frequencies were $B r s d_{1}-B r s d_{2}-b r s d_{3} b r s d_{3}$ (9/64), Brsd ${ }_{1}-$ brsd $_{2}$ brsd $_{2}$ brsd $_{3}$ brsd $_{3}$ (3/64), brsd $_{1}$ brsd $_{1}$ Brsd $_{2}-$ brsd $_{3}$ brsd $_{3}$ (3/64), brsd brsd $_{1}$ brsd $_{2}$ brsd $_{2}$ Brsd $_{3}$ Brsd $_{3}$ (3/64), and brsd brsd $_{1}$ brsd $_{2}$ brsd $_{2}$ brsd $_{3}$ brsd $_{3}$ (1/64). In the $\mathrm{BC}_{1} \mathrm{~F}_{1}$ generation, the Greyed-purple (183C) and the parental type Greyed-orange (167B/C) seeds were produced in equal $(1: 1)$ proportion. The genotypes $\operatorname{Brsd}_{1} \mathrm{Brsd}_{1} \mathrm{Brsd}_{2} \mathrm{Brsd}_{2} \mathrm{Brsd}_{3} \mathrm{brsd}_{3}, \mathrm{Brsd}_{1}$ $\operatorname{brsd}_{1} \mathrm{Brsd}_{2} \mathrm{Brsd}_{2} \mathrm{Brsd}_{3} \mathrm{brsd}_{3}, \quad \mathrm{Brsd}_{1} \mathrm{Brsd}_{1} \mathrm{Brsd}_{2} \mathrm{brsd}_{2}$ $\mathrm{Brsd}_{3} \mathrm{brsd}_{3}$, and $\mathrm{Brsd}_{1} \mathrm{brsd}_{1} \mathrm{Brsd}_{2} \mathrm{brsd}_{2} \mathrm{Brsd}_{3} \mathrm{brsd}_{3}$ produced Greyed-purple (183C) seeds; while the plants with the genotypes of $\operatorname{Brsd}_{1} \mathrm{Brsd}_{1} \mathrm{Brsd}_{2} \mathrm{Brsd}_{2} \mathrm{brsd}_{3}$ $\operatorname{brsd}_{3}, \mathrm{Brsd}_{1} \mathrm{Brsd}_{1} \mathrm{Brsd}_{2} \mathrm{brsd}_{2} \mathrm{brsd}_{3} \mathrm{brsd}_{3}, \mathrm{Brsd}_{1} \mathrm{brsd}_{1}$ $\mathrm{Brsd}_{2} \mathrm{brsd}_{2} \mathrm{brsd}_{3} \mathrm{brsd}_{3}$, and $\mathrm{Brsd}_{1} \mathrm{brsd}_{1} \mathrm{Brsd}_{2} \mathrm{Brsd}_{2}$ $\operatorname{brsd}_{3} \mathrm{brsd}_{3}$ were responsible for producing the parental type Greyed-orange (167B/C) seed color. The mode of inheritance of seed coat color in the hybrid 'ICPH 2671' is explained on the basis of a tri-genic model: $\mathrm{Brsd}_{1}-\mathrm{Brsd}_{2}-\mathrm{brsd}_{3} \mathrm{brsd}_{3}$ had Greyed-orange (167B/C) color seed; $\mathrm{Brsd}_{1}-\mathrm{Brsd}_{2}-\mathrm{Brsd}_{3}$ produced reddish Greyed-orange (167B/C) seeds. In this case the genes $\mathrm{Brsd}_{1} / \mathrm{Brsd}_{2}$ are complimentary, while $\mathrm{Brsd}_{3}$ acts as a modifier gene. Considering the segregation patterns in different generations it was postulated that the genetic constitution of 'ICPA 2043' is $\mathrm{Brsd}_{1} \mathrm{Brsd}_{1} \mathrm{Brsd}_{2}$ $\mathrm{Brsd}_{2} \mathrm{brsd}_{3} \mathrm{brsd}_{3}$ and the genotype of the restorer line is $\operatorname{brsd}_{1} \operatorname{brsd}_{1} \operatorname{brsd}_{2} \mathrm{brsd}_{2} \mathrm{Brsd}_{3} \mathrm{Brsd}_{3}$, while the resultant $\mathrm{F}_{1}$ hybrid had the genotypic constitution of $\mathrm{Brsd}_{1} \mathrm{Brsd}_{1} \mathrm{Brsd}_{2} \mathrm{Brsd}_{2} \mathrm{brsd}_{3} \mathrm{brsd}_{3}$.

\section{Conclusion}

To overcome the constraint of stagnant yield the first commercial pigeonpea hybrid 'ICPH 2671' was developed. In spite of its 30-35\% yield advantage in farmers' fields, its adoption is limited due to its greyed purple seed color which was not preferred by the consumers. Both the parents and their hybrid (crossed) seed have brown color while the commercial crop is greyed purple seeded that fetches about $10 \%$ less price in the market. Therefore it became essential to understand the genetics of seed color control in pigeonpea. The data revealed the presence of three independent dominant genes which controlled the expression of seed coat color. In the hybrid plants genes $\mathrm{Brsd}_{1}$ and $\mathrm{Brsd}_{2}$ act in complementation with the third gene $B r s d_{3}$, which also act as a modifier. The presence of $\mathrm{Brsd} 3$ gene in a genotype together with $\mathrm{Brsd}_{1}$ and/or $\mathrm{Brsd}_{2}$ produces a strong inter-allelic epistatic interactions resulting in the greyed-purple (183C) seed color. This study has also revealed that the genetic constitution female parent 'ICPA 2043' is Brsd $_{1}$ Brsd $_{1}$ Brsd $_{2}$ Brsd $_{2}$ brsd $_{3}$ brsd $_{3}$, while the genotype of the restorer line 'ICPR 2671' is $\operatorname{brsd}_{1} \operatorname{brsd}_{1} \mathrm{brsd}_{2} \mathrm{brsd}_{2} \mathrm{Brsd}_{3} \mathrm{Brsd}_{3}$.

Open Access This article is distributed under the terms of the Creative Commons Attribution Noncommercial License which permits any noncommercial use, distribution, and reproduction in any medium, provided the original author(s) and source are credited.

\section{References}

Kyu KhinLay, Saxena KB, Kumar RV, Rathore A (2011) Prospects of hybrids in enhancing production and 
productivity of pigeonpea in Myanmar. J Food Legum 24:1-7

Remanandan P (1990) Pigeonpea: genetic resources. In: Nene YL, Hall SD, Sheila VK (eds) The pigeonpea. CAB International, Wallingford, pp 89-115

Saxena KB (2008) Genetic improvement of pigeonpea-a review. Trop Plant Biol 1:159-178

Saxena KB (2010) Breeding hybrids for enhancing productivity in pigeonpea. In: Rao SK, Kallo G, Khare D (eds)
Promotion of public-bred hybrids of field crops. Studium Press, India, pp 155-163

Saxena KB, Sharma D (1990) Pigeonpea genetics. In: Nene YL, Hall SD, Sheila VK (eds) The pigeonpea. CAB International, Wallingford, pp 137-158

Saxena KB, Kumar RV, Srivastava N, Shying B (2005) A cytoplasmic-genic male-sterility system derived from a cross between Cajanus cajanifolius and Cajanus cajan. Euphytica 145:291-296 